\title{
On the Computation of Finite Invariant Sets of Mappings*
}

\author{
By Alex Gelman and Werner C. Rheinboldt \\ Dedicated to Professor Eugene Isaacson on the occasion of his 70th birthday
}

\begin{abstract}
This paper suggests a new computational method for determining closed curves that are invariant under a given mapping. Unlike other authors, we discretize not only the curve but also the mapping itself. This allows us to avoid completely the computational difficulties connected with the numerical solution of large linear systems. The method uses simple recurrence formulas, which greatly reduce the execution times.
\end{abstract}

The problem of determining closed curves that are invariant under some given mapping arises in many applications, and various numerical techniques have been proposed for the calculation of such invariant cycles. We refer here only to Doedel [1], Iooss et al. [3], Kevrekidis et al. [4], van Veldhuizen [6], where further references may be found.

As in [4], we consider a continuously differentiable mapping

$$
(s, x) \in S^{1} \times R^{m} \rightarrow(\Phi(s), F(s, x)), \quad \Phi: S^{1} \rightarrow S^{1}, \quad F: S^{1} \times R^{m} \rightarrow R^{m},
$$

where $S^{1}$ is the unit circle in $R^{2}$, parametrized by $s$. In other words, (1) is a mapping of the cylinder $S^{1} \times R^{m}$ into itself. Then

$$
\gamma: S^{1} \rightarrow S^{1} \times R^{m}, \quad \gamma(s)=(s, r(s)), \quad s \in S^{1},
$$

is invariant under (1) exactly if

$$
r(\Phi(s))=F(s, r(s)), \quad s \in S^{1} .
$$

As usual, for the computation we approximate this curve by some polygon with vertices $x_{i} \approx r\left(s_{i}\right), i=1,2, \ldots, n$. But, other than in the cited references, we also discretize the circle mapping $\Phi$ by the following mapping from $N=\{1,2, \ldots, n\}$ into itself:

$$
\Psi: N \rightarrow N, \quad \Psi(i)=\left[\left\{\frac{\Phi\left(s_{i}\right)}{2 \pi}\right\} n\right], \quad i \in N .
$$

Here, $[a]$ denotes the closest integer to $a \in R^{1}$ and $\{a\}$ the nonnegative fractional part of $a$. Then, our discretization of (3) has the form

$$
x_{\Psi(i)}=F\left(s_{i}, x_{i}\right), \quad i \in N,
$$

Received March 7, 1988.

1980 Mathematics Subject Classification (1985 Revision). Primary 14E99, 39-04, 39B30, 65D20.

* This work was supported in part by the Office of Naval Research under contract N-00014-80C-9455, the National Science Foundation under grant DCR-8309926, and the Air Force Office of Scientific Research under grant 84-0131. 
and hence represents a problem of finding a finite invariant set

$$
P=\left\{\left(s_{1}, x_{1}\right), \ldots,\left(s_{n}, x_{n}\right)\right\}
$$

of (1).

For the numerical solution of discretizations of the problem (3), Newton's method is frequently employed. But then the computational cost is typically of the order of $(m n)^{3}$ and hence grows quickly with the number of vertices of the approximating polygon. Here we want to show that for discretizations of the form (5) it is possible to achieve a growth rate that is linear in $m n$. This is certainly a considerable reduction, especially for applications involving periodic solutions of large systems of ordinary differential equations.

Let $\Gamma$ denote the connectivity graph representing the mapping $\Psi: N \rightarrow N$ of (4); that is, $\Gamma$ has $N$ as its vertex set and a directed arc from $i \in N$ to $j \in N$ exactly if $j=\Psi(i)$. Since $\Psi$ is a mapping of $N$ into itself, the out-degree of any vertex of $\Gamma$ is exactly one, and, for any starting point $i \in N$, the directed path through the vertices

$$
i_{0}=i, \quad i_{k+1}=\Psi\left(i_{k}\right), \quad k=0,1, \ldots,
$$

is uniquely determined. Because $\Gamma$ has only $n$ vertices, any such path ultimately involves repeated vertices. Suppose that the first $q+1$ vertices $i_{k}, k=0, \ldots, q$, of (7) are distinct, but that $i_{q+1}$ repeats one of these earlier vertices. Then we have two possible cases, namely,

(i) $i_{q+1}=i_{q}$; that is $i_{q}$ is a fixed point of $\Psi$ which implies that $i_{k}=i_{q}$ for $k \geq q$.

(ii) $i_{q+1}=i_{p}$ for some $p, 0 \leq p<q$. Then the vertices $i_{k}, k=p, p+1, \ldots, q$, form a nondegenerate, directed cycle of $\Gamma$.

If two paths (7) with distinct starting points have a vertex in common, then they must be identical from then on, for otherwise there would have to be a vertex with out-degree two. Hence, in particular, no two distinct cycles can intersect. Thus the vertices $i$ of $\Gamma$ belong to three disjoint classes:

(a) $i$ is a fixed point of $\Psi$,

(b) $i$ belongs to a unique cycle of $\Gamma$,

(c) there is a unique directed path through $i$ which terminates either at a fixed point of $\Psi$ or on a cycle of $\Gamma$.

In other words, $\Gamma$ consists of finitely many disjoint components $\Gamma_{j}, j=1, \ldots, r$, each of which contains exactly one fixed point of $\Psi$ or one nondegenerate cycle of $\Gamma$, and all other vertices of $\Gamma_{j}$ belong to the class (c).

If Newton's method is applied to the system (5), then, at each step, a linear system of the form

$$
(V-A) u=b, \quad u=\left(u_{1}, \ldots, u_{n}\right)^{T}, \quad u_{i} \in R^{m}
$$

has to be solved, where

$$
V u=\left(\Psi\left(u_{1}\right), \ldots, \Psi\left(u_{n}\right)\right)^{T}
$$

and

$$
\begin{gathered}
A=\operatorname{diag}\left(A_{1}, \ldots, A_{n}\right), \quad A_{i}=D F\left(x_{i}\right) \in R^{m \times m} \\
b=\left(b_{1}, \ldots, b_{n}\right)^{T}, \quad b_{i}=F(x(i)) \in R^{m}
\end{gathered}
$$


Evidently, (8) represents a recursion

$$
u_{i_{k+1}}=b_{i_{k}}+A_{i_{k}} u_{i_{k}}, \quad i_{k+1}=\Psi\left(i_{k}\right), \quad k=0,1,2, \ldots
$$

Thus, for any vertex $i$ of $\Gamma$ of class (a), that is, for any fixed point $i$ of $\Psi$, the value of $u_{i}$ can be calculated from

$$
\left(I_{m}-A_{i}\right) u_{i}=b_{i}
$$

where $I_{m}$ is the identity on $R^{m}$, if only the matrix on the left is nonsingular. Let $i$ be some vertex of $\Gamma$ of class (b) or (c). Then the path (7) started at $i_{0}=i$ begins with $q+1$ distinct vertices $i_{k}, k=0,1, \ldots, q$, and we have $i_{q+1}=i_{p}$ for some $p$ with $0 \leq p \leq q$.

We define recursively the quantities

$$
d_{i_{k+1}}=b_{i_{k}}+A_{i_{k}} d_{i_{k}}, \quad B_{i_{k+1}}=A_{i_{k}} B_{i_{k}}, \quad k=0,1, \ldots, q ; d_{i_{0}}=0, B_{i_{0}}=I
$$

then (9) can be rewritten in terms of the unknown first $u$-value $u_{i_{0}}$,

$$
u_{i_{k}}=d_{i_{k}}+B_{i_{k}} u_{i_{0}}, \quad k=0,1, \ldots, q .
$$

Therefore, $u_{i_{0}}$ can be computed from the equation

$$
\left(B_{i_{p}}-B_{i_{q}}\right) u_{i_{0}}=d_{i_{q}}-d_{i_{p}}
$$

provided that the difference of the $B$-matrices on the left is nonsingular. Then, in turn, all $u_{i_{k}}, k=1,2, \ldots, q$ can be calculated from (11). Moreover, for any other vertex in the same component of $\Gamma$ as $i_{0}$, the corresponding path will end at a vertex $j$ where the $u$-value is already known. Hence, from (12) we can calculate $u_{j}$ provided that $B_{j}$ is invertible. Thus, by continuing this process, we can compute the $u$-values at all vertices of $\Gamma$ as long as no singular matrices are encountered. Since this approach is equivalent with a recursive evaluation of the solution of (8), it follows readily that -in real arithmetic-no singular matrix will ever arise if the matrix $V-A$ in (8) is nonsingular.

Evidently, this recursive solution of (8) involves only the solution of linear systerns of the order $m$, and hence can be expected to be considerably faster than any standard decomposition of the full matrix in (8). In fact, all our examples show that the run-time of the process is approximately linear in $\mathrm{nm}$.

In many applications, the circle map $\Phi$ in (1) is a fixed rotation:

$$
s \rightarrow s+\omega \bmod 2 \pi, \quad 0<\omega<2 \pi .
$$

Here it follows directly from (4) that $\Psi$ must be injective, and hence represents a permutation on $N$. Thus, $\Gamma$ now consists of a finite number of disjoint cycles, and the solution of (8) involves only the solution of finitely many systems of the form (12).

As a first sample problem for this case, we consider the following function (1), used also in [4]:

$$
\begin{gathered}
\Phi(s)=s+\omega \bmod 2 \pi, \quad \omega=2 \pi\left(5^{1 / 2}-1\right), \quad s \in S^{1}, \\
F(s, x)=\lambda x(1-x)+\varepsilon \cos (s), \quad s \in S^{1}, x \in R^{1},
\end{gathered}
$$

where $\lambda=3.46$ and $\varepsilon$ is a parameter. 
A code was implemented on a VAX- 8650 which incorporates the above solution method. It uses a continuation procedure similar to that described in [5] to obtain invariant cycles for a sequence of $\varepsilon$-values starting from the constant solution $x=$ 0.44 for $\varepsilon=0.001$. Table 1 gives some of the computed values of $r(0)$ for different $\varepsilon$ and for $n=377$.

\begin{tabular}{ll}
\multicolumn{2}{c}{ TABLE 1} \\
\hline \multicolumn{1}{c}{$\varepsilon$} & $r(0)$ \\
\hline 0.001 & 0.437523 \\
0.01 & 0.411982 \\
0.02 & 0.384902 \\
0.03 & 0.376986 \\
0.032 & 0.375482 \\
0.034 & 0.373925 \\
0.035 & 0.375158 \\
0.0351 & 0.374768 \\
0.0352 & 0.374059 \\
0.035245 & 0.373697 \\
\hline
\end{tabular}

All calculations used double precision and a corrector tolerance of $10^{-6}$. A corresponding run with $n=610$ produced values of $r(0)$ which differed only by about $1.0 \times 10^{-5}$ from those in Table 1. The elapsed clock-time $T$ for the execution of each cycle was recorded, and Table 2 shows the quotients $T /(n k)$ for different dimensions $n$, where $k$ denotes the number of Newton corrector steps taken by the process. The linear dependence of the time on $n$ is clearly visible.

TABLE 2

\begin{tabular}{cccc}
\hline$n$ & 233 & 377 & 610 \\
\hline$\varepsilon$ & $\frac{T}{n k}$ & $\frac{T}{n k}$ & $\frac{T}{n k}$ \\
\hline 0.001 & 2.58 & 3.24 & 4.36 \\
0.002 & 2.58 & 3.24 & 4.36 \\
0.003 & 2.58 & 3.27 & 4.37 \\
\hline
\end{tabular}

For a second class of problems, we consider a system of ordinary differential equations

$$
x^{\prime}=f(x, t), \quad x \in R^{m}, t \in R^{1},
$$

which is assumed to have a $2 \pi$-periodic solution $x_{0}(t)$. If $x_{i} \approx x_{0}\left(t_{i}\right), i \in N$, denotes some approximation of this solution, then the backward Euler discretization

$$
x_{i+1}=x_{i}+h f\left(x_{i+1}, t_{i+1}\right)
$$

represents a system of the form (5) with a circle mapping (14a) for which $\omega=1$. Hence we can apply the recursive solution process to this system.

As a first sample problem, we consider the following simple form of van der Pol's equation:

$$
x^{\prime \prime}-\lambda\left(1-x^{2}\right) x^{\prime}+x=0 .
$$


As shown, for example in [2], the solution satisfies

$$
x=2 \cos (\omega t)+\lambda(0.75 \sin (\omega t)-0.25 \sin (3 \omega t))+O\left(\lambda^{2}\right) \quad \text { as } \lambda \rightarrow 0,
$$

and $\omega=1+O\left(\lambda^{2}\right)$ as $\lambda \rightarrow 0$.

In Table 3 we give some of the results of a calculation with the above-mentioned code for this problem when $\omega=1$.

TABLE 3

\begin{tabular}{|c|c|c|c|c|c|c|c|c|}
\hline & $n=64$ & & $n=128$ & & $n=256$ & & $n=512$ & \\
\hline$\lambda$ & err & $\frac{T}{n k}$ & err & $\frac{T}{n k}$ & err & $\frac{T}{n k}$ & err & $\frac{T}{n k}$ \\
\hline 0.02 & 0.01654 & 3.91 & 0.01560 & 3.70 & 0.01524 & 3.61 & 0.01511 & 3.55 \\
\hline 0.04 & 0.03358 & 4.06 & 0.03162 & 3.71 & 0.03081 & 3.63 & 0.03050 & 3.54 \\
\hline 0.06 & 0.05112 & 4.06 & 0.04811 & 3.83 & 0.04684 & 3.63 & 0.04631 & 3.57 \\
\hline 0.08 & 0.06917 & 4.22 & 0.06522 & 3.75 & 0.06345 & 3.63 & 0.06271 & 3.57 \\
\hline 0.10 & 0.08777 & 4.22 & 0.08292 & 3.83 & 0.08073 & 3.67 & 0.07979 & 3.56 \\
\hline
\end{tabular}

Here, err denotes the error in the infinity norm of $R^{n}$ between the computed derivatives $x^{\prime}$ and the $x^{\prime}$-values obtained from the expansion (17). The corresponding errors in the $x$-values are slightly smaller. Evidently, the error due to the zero-order approximation of the frequency $\omega$ dominates the discretization error. Once again, the time depends linearly on $n$.

As a second problem, we used the periodically forced brusselator

$$
x^{\prime}=A+x^{2} y-B x-x+a \cos \omega t, \quad y^{\prime}=B x-x^{2} y .
$$

Here, $a$ and $\omega$ are parameters and a frequent choice for the constants $A$ and $B$ is $A=0.4, B=1.2$. The unforced brusselator $(a=0)$ then has a limit cycle with the natural frequency $\omega_{0}=0.3750375$.

For our computations we considered the resonance case $\omega=\omega_{0}$. Then, for $0<|a| \leq a_{\text {crit }}=0.0033$, three periodic solutions are obtained, two of which are inside the original limit cycle and one outside. With increasing $|a|$, the inside solutions approach each other, until for $|a|=a_{\text {crit }}$ they coincide and for $|a|>a_{\text {crit }}$ they disappear. The amplitude of the outside solution increases with $|a|$.

In order to obtain suitable starting solutions for the continuation process along these three solution branches, we imbed (18) into a homotopy depending on a new parameter $\lambda$. For this, the equations are first transformed to the new time $t \rightarrow \omega_{0} t$. Then, by adding them, we see that $z=x+y$ satisfies

$$
z^{\prime}=\tau(A-x-a \cos t), \quad \tau=1 / \omega_{0} .
$$

Now $x, x^{\prime}$ are linear expressions of $z^{\prime}, z^{\prime \prime}$, and by substituting them into the transformed equations and eliminating $x$ and $y$, we obtain for $u=z-(A+B / A)$ the equation

$$
\begin{aligned}
u^{\prime \prime} & =-f\left(u, u^{\prime}, t, \omega\right)-u\left(A \tau-u^{\prime}\right)^{2}+u^{\prime 2}(2 A-B / A), \\
(19 \mathrm{~b}) \quad f(u, v, t, \omega) & =\tau\left(1+A^{2}-B\right) v+\omega v^{3}+a \tau^{2} \cos t+a \tau\left(A \tau-u^{\prime}\right)^{2} \sin t .
\end{aligned}
$$


From this, the desired homotopy is constructed as follows

$$
u^{\prime}=v, \quad v^{\prime}=-\lambda f(u, v, t, \omega(\lambda))-u\left(A \tau-\lambda^{2} v\right)^{2}+\lambda^{2} v^{2}(2 A-B / A)
$$

where $\omega(\lambda)=A-0.0249625 \lambda$. Clearly, for $\lambda=1$ this system is equivalent with $(19 \mathrm{a} / \mathrm{b})$, and for $\lambda=0$ it reduces to the system $u^{\prime}=v, v^{\prime}=-u$, which has an infinite set of $2 \pi$-periodic solutions. The limit of the periodic solutions of (20) for $\lambda \rightarrow 0$ can be derived by the classical small-parameter technique. For this, we need to solve the equations

$$
\int_{0}^{2 \pi} f\left(u_{0}, v_{0}, t, 0\right) \sin t d t=0, \quad \int_{0}^{2 \pi} f\left(u_{0}, v_{0}, t, 0\right) \cos t d t=0
$$

with

$$
u_{0}=\alpha \sin t+\beta \cos t, \quad v_{0}=\alpha \cos t-\beta \sin t .
$$

For $\omega(0)=A$, the evaluation of these integrals produces the system

$$
\begin{gathered}
\left(1+A^{2}-B\right) \beta-0.75 A^{2} \beta\left(\alpha^{2}+\beta^{2}\right)+a=0 \\
\left(1+A^{2}-B\right) \alpha-0.75 A^{2} \alpha\left(\alpha^{2}+\beta^{2}\right)+a / A=0
\end{gathered}
$$

which has the solutions

$$
\alpha=C \gamma, \quad \beta=-A \alpha, \quad C=\frac{2}{A}\left(\frac{B-1-A^{2}}{3\left(1+A^{2}\right)}\right)^{1 / 2},
$$

where $\gamma$ satisfies

$$
\gamma^{3}-\gamma+D=0, \quad D=\frac{4 a}{3(A C)^{3}\left(1+A^{2}\right)}
$$

This equation for $\gamma$ has three real roots for $|D| \leq 2 / 3^{4 / 3}$, that is, for

$$
|a| \leq a_{\text {crit }}=\frac{2 a}{3^{4 / 3} D} \approx 0.0033 \text {. }
$$

Exactly one of these roots has a different sign than $a$ and always exceeds 1 in modulus. The other two roots have the same sign as $a$ and are less than one in modulus; moreover, they coincide for $|a|=a_{\text {crit }}$ and become complex for $|a|>a_{\text {crit }}$.

For the computation, we use now the homotopy (20) with $a=0$, in which case the three roots of $(21)$ are $\gamma=-1,0,1$. With the corresponding functions $u_{0}, v_{0}$ for one of these roots as starting point, we begin the continuation process from $\lambda=0$ and proceed until at $\lambda=1$ some functions $u_{1}, v_{1}$ are obtained. They, in turn, become the starting point for the continuation process in the parameter $a$, beginning from $a=0$ and with $\lambda$ held at the fixed value 1 . During both continuations it is advisable to introduce in (21) the transformed variables $u \rightarrow u-u_{j}, v \rightarrow v-v_{j}$ with $j=0,1$, respectively.

In terms of the original variables $x, y$ of (18), the second continuation process produces points along a branch of periodic solutions of the forced brusselator. The properties of these solutions depend on the value of $\gamma$ with which the first continuation was started. More specifically, as expected, in the two cases $\gamma=-1,0$ these solutions cease to exist when $|a|$ exceeds $a_{\text {crit }}$, while for $\gamma=1$ our second continuation process did not fail. 
We present here only some results for the latter case $\gamma=1$. The first continuation always proceeded with steps of order 0.1 from $\lambda=0$ to $\lambda=1$. In Table 4 we give some results for the second continuation with $n=64$.

TABLE 4

\begin{tabular}{cccccc}
\hline$a$ & $r$ & $\frac{T}{n k}$ & $a$ & $r$ & $\frac{T}{n k}$ \\
\hline 0.00 & 0.4561 & 4.22 & 0.11 & 0.3938 & 4.30 \\
0.01 & 0.4292 & 4.18 & 0.12 & 0.3865 & 4.38 \\
0.02 & 0.3981 & 4.32 & 0.13 & 0.3579 & 4.38 \\
0.03 & 0.3604 & 4.38 & 0.14 & 0.3363 & 4.32 \\
0.04 & 0.3119 & 4.34 & 0.15 & 0.3216 & 4.32 \\
0.05 & 0.2399 & 4.22 & 0.20 & 0.2960 & 4.42 \\
0.06 & 0.08461 & 4.48 & 0.25 & 0.2971 & 4.38 \\
0.07 & 0.02140 & 4.22 & 0.30 & 0.3070 & 4.48 \\
0.08 & 0.03430 & 4.30 & 0.35 & 0.3224 & 4.38 \\
0.09 & 0.09779 & 4.42 & 0.40 & 0.3424 & 4.61 \\
0.10 & 0.1986 & 4.30 & & & \\
\hline
\end{tabular}

Here, $r=\left(x(0)^{2}+x^{\prime}(0)^{2}\right)^{1 / 2}$, and not all continuation steps are shown. In fact, the process slows down before the turning points of $r$. The linear dependence of the time on $n$ is again evident.

Department of Mathematics and Statistics

University of Pittsburgh

Pittsburg, Pennsylvania 15260

E-mail: wcr@pitt.csnet

1. E. DOEDEL, Software for Continuation and Bifurcation Problems in Ordinary Differential Equations, California Institute of Technology, Applied Mathematics Report 1986.

2. C. HAYAShI, Nonlinear Oscillations in Physical Systems, Princeton Univ. Press, Princeton, N. J., 1964.

3. G. Iooss, A. Arneodo, P. Coullet w כ. Tresser, Simple Computation of Bifurcating Invariant Circles for Mappings, Lecture Notes in Math., Vol. 898, Springer-Verlag, Berlin and New York, 1981, pp. 192-211.

4. I. G. KeVRekidis, R. ARIS, L. D. SChmidt \& S. PELIKAN, "Numerical computation of invariant circles of maps," Phys. D, v. 16, 1985, pp. 243-251.

5. W. C. RHEINBOLDT \& J. V. BURKARDT, "A locally parametrized continuation process," ACM Trans. Math. Software, v. 9, 1983, pp. 215-235.

6. M. VAN VELDHUIZEN, "Convergence results for invariant curve algorithms," Math. Comp., v. 51,1988 , pp. $677-697$. 